Check for updates

Cite this: Phys. Chem. Chem. Phys., 2020, 22, 3734

Received 29th November 2019, Accepted 21st January 2020

DOI: $10.1039 / \mathrm{c} 9 \mathrm{cp} 06468 \mathrm{k}$

rsc.li/pccp

\title{
Stability of the chaperonin system GroEL-GroES under extreme environmental conditions $\dagger$
}

\author{
Michel W. Jaworek, Simone Möbitz, Mimi Gao and Roland Winter (D)*
}

\begin{abstract}
The chaperonin system GroEL-GroES is present in all kingdoms of life and rescues proteins from improper folding and aggregation upon internal and external stress conditions, including high temperatures and pressures. Here, we set out to explore the thermo- and piezostability of GroEL, GroES and the GroELGroES complex in the presence of cosolvents, nucleotides and salts employing quantitative FTIR spectroscopy and small-angle X-ray scattering. Owing to its high biological relevance and lack of data, our focus was especially on the effect of pressure on the chaperonin system. The experimental results reveal that the GroEL-GroES complex is remarkably temperature stable with an unfolding temperature beyond $70{ }^{\circ} \mathrm{C}$, which can still be slightly increased by compatible cosolutes like TMAO. Conversely, the pressure stability of GroEL and hence the GroEL-GroES complex is rather limited and much less than that of monomeric proteins. Whereas GroES is pressure stable up to $\sim 5 \mathrm{kbar}$, GroEl and the GroEl-GroES complex undergo minor structural changes already beyond $1 \mathrm{kbar}$, which can be attributed to a dissociation-induced conformational drift. Quite unexpectedly, no significant unfolding of GroEL is observed even up to $10 \mathrm{kbar}$, however, i.e., the subunits themselves are very pressure stable. As for the physiological relevance, the structural integrity of the chaperonin system is retained in a relatively narrow pressure range, from about 1 to 1000 bar, which is just the pressure range encountered by life on Earth.
\end{abstract}

\section{Introduction}

Chaperonins represent an important class of proteins in biological organisms (archaea, bacteria and eukarya), which have an essential role in assisting protein folding by transient encapsulation of nascent proteins in an ATP-driven mechanism to prevent misfolding or aggregation. Moreover, stress-induced denaturation of proteins can be counteracted by the chaperonin's support of correct refolding. ${ }^{1-4}$ Here, we investigated the effect of temperature and pressure stress on the conformational stability of the heat shock protein (Hsp) complex GroELGroES of Escherichia coli (Fig. 1a), which belongs to the group I chaperonins. Chaperonins, like the GroEL-GroES complex, are oligomeric proteins, which consist of a large double-ring structure stacked back to back, thereby enclosing a central cavity with its lid-like cofactor. In this case, the lid-like cofactor refers to GroES, which contains seven identical $\sim 10 \mathrm{kDa}$ subunits assembled as a heptamer ring and caps the ends of the GroEL cylinder. Two heptameric rings of GroEL (each subunit contains $\sim 57 \mathrm{kDa}$ ) form the cavity, exposing hydrophobic amino

Physical Chemistry I - Biophysical Chemistry, Faculty of Chemistry and Chemical Biology, Technical University Dortmund, Otto-Hahn-Straße 4a, 44227 Dortmund, Germany. E-mail: roland.winter@tu-dortmund.de; Tel: +49231755 3900 $\dagger$ Electronic supplementary information (ESI) available. See DOI: 10.1039/ c9cp06468k acid residues for the binding of folding intermediates. Each subunit can be divided into an apical, intermediate and equatorial domain that feature different functions during the reaction cycle. ${ }^{1,2,5}$ In the first step of the reaction cycle (Fig. 1b), the non-native protein binds to the free end of a GroEL-GroES complex. The ATP-mediated conformational rearrangement of each subunit of GroEL (light green) and the binding of GroES leads to the encapsulation. Within the chaperonin nanocage, the folding takes place for the time required for ATP hydrolysis ( 2-10 s) on each subunit of the heptameric ring (pink). ATP binding to the opposite ring triggers the release of GroES and the folded protein, which completes the protein folding cycle of the chaperonin system. In case of an incomplete folding of the protein, it will rebind after release. ${ }^{1,2,4-7}$

Besides the "quality control" during the translation process of proteins, chaperonins help to properly refold denatured proteins. But what about the stability of the chaperonin system itself under extreme environmental conditions? A series of biochemical and structural studies have been carried out on the complex and its components looking into the effects of salts, ligands and temperature. ${ }^{7-12}$ Almost nothing is known about the effect of pressure on the stability of the chaperonin system despite the fact that a huge fraction of organisms in the global biosphere is thriving at high hydrostatic pressure (HHP) conditions in the deep sea and sub-sea floor, where pressures of 1000 bar and more can be reached. ${ }^{13-16}$ 
a)

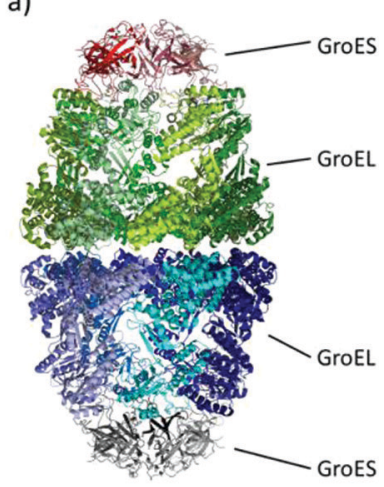

b)

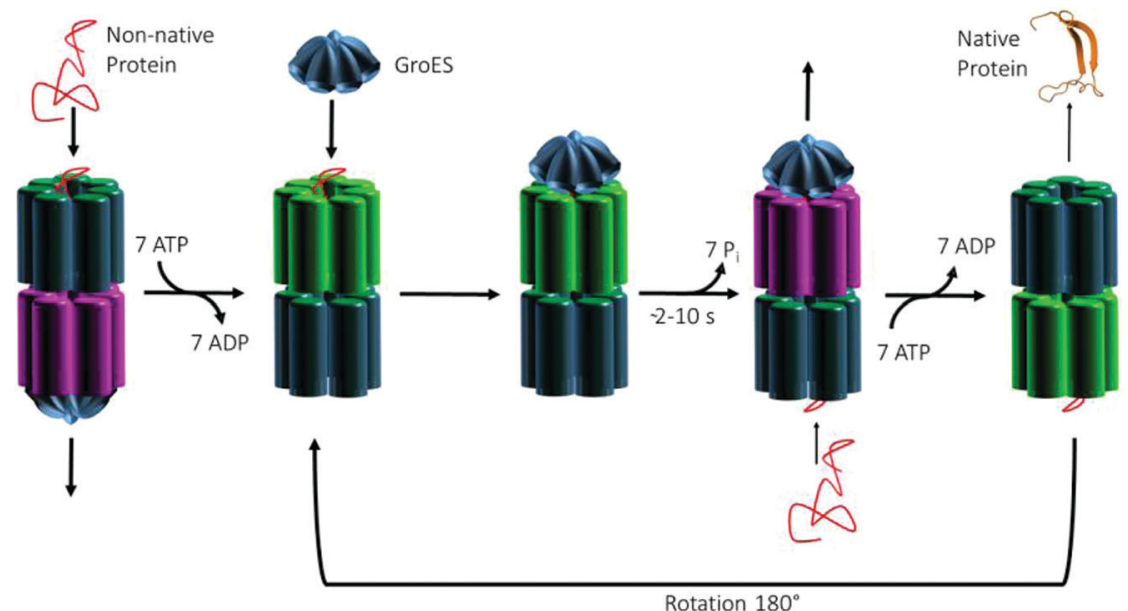

Fig. 1 (a) The GroEL-GroES complex in secondary structure representation (PDB: 3WVL), (b) Schematic of the reaction cycle of the GroEL-GroES system.

In fact, marine sediments have been shown recently to represent one of the largest microbial habitats on Earth, even down to about $2 \mathrm{~km}$ below the ocean floor, where next to high pressures also increasing temperatures are encountered. ${ }^{15}$ Interestingly, a relatively high concentration of particular osmolytes, such as trimethylamine- $N$-oxide (TMAO), was found in cells of deep sea organisms, which are thought to help rescuing cells from pressure-induced deterioration, including protein unfolding at high hydrostatic pressures. ${ }^{17-20}$ Hence, HHP studies on biomolecular systems are prerequisite for understanding current and ancient life in the deep sea, an environment which is also the potential birth place of life on Earth. ${ }^{13,14}$

Remarkably, very little is known about the temperature and pressure stability of the chaperonin system and the effect of cosolutes on its stability. What is known, however, is that the expression level in $E$. coli is not only upregulated by temperature stress, but also under high pressure. ${ }^{13,14}$ Horowitz et al. explored the dissociation of GroEL and GroES in the presence of $\mathrm{Mg}^{2+}$ and nucleotides as a function of pressure using lightscattering data, indicating dissociation around $2 \mathrm{kbar}^{21-23}$ Furthermore, different chaotropic cosolvents, like urea or guanidinium hydrochloride, have revealed an adverse influence on the stability of both proteins. ${ }^{24}$ In this study, we set out to explore the thermo- and piezostability of GroEL, GroES and the GroEL-GroES complex in the presence of cosolvents, nucleotides and salts in more detail employing quantitative Fourier-transform infrared (FTIR) spectroscopy, complemented by small-angle X-ray scattering (SAXS).

\section{Materials and methods}

\subsection{Chemicals and sample preparation}

GroEL and GroES genes were produced using the pET-11a vector, cloned in $E$. coli BL21(DE3)-Codon plus RIPL competent cells from (Agilent Technologies) in LB-medium containing ampicillin and chloramphenicol and incubated overnight.
Upon reaching an $\mathrm{OD}_{600 \mathrm{~nm}}$-value of 0.5 , protein expression was induced with $1 \mathrm{mM}$ IPTG (isopropyl $\beta$-D-1-thiogalactopyranoside). After 3 hours, the cells were centrifuged at $4{ }^{\circ} \mathrm{C}$ and $6000 \mathrm{rpm}$. The cell pellets obtained were resuspended in purification buffer (50 mM Tris/HCl buffer, $\mathrm{pH}$ 7.5, $20 \mathrm{mM}$ $\mathrm{NaCl}, 1 \mathrm{mM}$ EDTA (ethylenediaminetetraacetic acid) and $1 \mathrm{mM}$ DTT (dithiothreitol)). Subsequently, protease inhibitor, DNase und lysozyme was added to the solution, sonicated and then centrifuged at $25000 \mathrm{rpm}$ and $10{ }^{\circ} \mathrm{C}$. For purification of GroEL, the supernatant was added to a HiTrapQHP column (GE Healthcare), washed with $12 \%$ purification buffer containing $1 \mathrm{M} \mathrm{NaCl}$ and finally eluted over $150 \mathrm{~mL}$ with a linear gradient from $0 \%$ to $100 \%$ (purification buffer containing $1 \mathrm{M} \mathrm{NaCl}$ ). Using SDS-PAGE, the protein was identified, fractions were pooled and added to the GeFi Superdex 75 26/600, which was equilibrated with purification buffer containing $250 \mathrm{mM} \mathrm{NaCl}$. GroEL was re-identified via SDS-PAGE, fractions were collected and frozen at $-80{ }^{\circ} \mathrm{C}$. The purification of GroES was carried out by adding the supernatant to the HiTrapQHP column, washed with $5 \%$ purification buffer containing $0.5 \mathrm{M} \mathrm{NaCl}$ and finally eluted over $150 \mathrm{~mL}$ with a linear gradient from $0 \%$ to $100 \%$ (purification buffer containing $0.5 \mathrm{M} \mathrm{NaCl}$ ). Protein purity was verified by SDS-PAGE, fractions were pooled and added to the GeFi Superdex 75 26/600, which was equilibrated with purification buffer containing $250 \mathrm{mM} \mathrm{NaCl}$. Then, GroES was re-identified via SDS-PAGE, fractions were collected and also frozen at $-80{ }^{\circ} \mathrm{C}$. Under physiological conditions, GroEL (14mer) and GroES (heptamer) only form the asymmetric complex despite the higher concentration of GroES. For H/D-exchange, which is needed for the FTIR spectroscopic studies, the proteins were dialysed against $\mathrm{D}_{2} \mathrm{O}$ using Amicon Ultra $(2 \mathrm{~mL})$ centrifugation units with $10 \mathrm{kDa}$ cutoff and subsequently lyophilized. Considering the effect of temperature or pressure on the $\mathrm{pH}$, $50 \mathrm{mM}$ phosphate buffer or $50 \mathrm{mM}$ Tris buffer was chosen for the FTIR measurements, respectively. Different from phosphate buffer, Tris buffer is suitable for pressure experiments because of its small $\mathrm{p} K_{\mathrm{a}}$ dependence upon hydrostatic pressurization. 
Both buffers have a pD-value of 7.5, which was adjusted by adding DCl or NaOD. Here, buffer $\mathrm{A}$ is the neat buffer system, while buffer B contains additional $100 \mathrm{mM} \mathrm{KCl}$. All chemicals were purchased from Sigma Aldrich and were used without further purification. For the FTIR measurements, anhydrous trimethylamine $\mathrm{N}$-oxide (TMAO) and isotope labeled ${ }^{13} \mathrm{C}$-urea were used to avoid overlapping of the amide I' band with the IR absorption of the deformation (OD)-band of water or the asymmetric $\nu(\mathrm{CO})$-band of urea.

\subsection{Temperature- and pressure-dependent FTIR spectroscopy}

Temperature-dependent Fourier-transform infrared (FTIR) spectra were collected in a Nicolet 5700 (Thermo Fisher Scientific) equipped with a liquid-nitrogen cooled MCT-detector (HgCdTe) in the wavenumber range between 4000 to $1100 \mathrm{~cm}^{-1}$. A sample volume of about $15 \mu \mathrm{L}$ was placed in the IR cell between two $\mathrm{CaF}_{2}$ windows, which is separated by a mylar spacer of $50 \mu \mathrm{m}$ thickness. An external, circulating water thermostat controlled the required temperature in the cell with an accuracy of $\pm 0.1{ }^{\circ} \mathrm{C}$. To ensure that the sample was equilibrated, each temperature was maintained for $\sim 10 \mathrm{~min}$ before collecting the spectra. Pressure-dependent FTIR measurements were performed using a MAGNA 550 (Thermo Fisher Scientific) equipped with a liquidnitrogen cooled MCT-detector (HgCdTe) in the wavenumber range between 4000 to $650 \mathrm{~cm}^{-1}$ and a temperature of $25{ }^{\circ} \mathrm{C}$. To achieve pressures up to $12 \mathrm{kbar}(1.2 \mathrm{GPa})$, a membrane-driven diamond anvil cell (Diacell ${ }^{\circledR}$ VivoDac, Almax easyLab) with type IIa diamonds (Almax easyLab), equipped with an automated pneumatic pressure controller (Diacell ${ }^{\mathbb{R}}$ iGM Controller, Almax easyLab), was used. A $50 \mu \mathrm{m}$ thick gasket of brass with $0.5 \mathrm{~mm}$ drilling was placed between two diamonds, holding $2.5 \mu \mathrm{L}$ of the sample (effective sample volume: $9.8 \mathrm{~nL}$ ). Using the pressuredependent stretching vibration of $\mathrm{SO}_{4}{ }^{2-}\left(983.5 \mathrm{~cm}^{-1}\right.$ at 1 bar $)$, $\mathrm{BaSO}_{4}$ was used as an internal pressure standard. ${ }^{25}$ A protein concentration of $15 \mathrm{mg} \mathrm{mL}^{-1}$ was used for all FTIR experiments. To avoid $\mathrm{CO}_{2}$ and humidity, the sample chamber was continuously purged with $\mathrm{CO}_{2}$-free and dry air. After placing the sample inside the cell, the waiting time was $30 \mathrm{~min}$ to ensure a good signal-to-noise ratio. For each temperature or pressure, spectra were averaged over 128 scans in a row and were processed with Happ-Genzel apodization using the Omnic 7.2 spectral processing software. The resolution was set at $2 \mathrm{~cm}^{-1}$. Spectra were processed and analyzed with Thermo Grams 8.0 software as follows: after buffer subtraction and smoothing, the area of the amide $\mathrm{I}^{\prime}$ band (1700-1600 $\mathrm{cm}^{-1}$ ) was normalized. To identify the number and position of the subbands and to detect conformational changes, Fourier self-deconvoluted (FSD) and 2nd derivative spectra were analyzed. The amide $\mathrm{I}^{\prime}$ band region of GroEL and GroES can be decomposed into seven subbands. To determine the relative changes in the population of secondary structure elements, mixed Gaussian-Lorentzian line shape functions were used in the linear combination fitting procedure. ${ }^{26}$ Based on the assumption that a two-state unfolding or dissociation process of the protein occurs, a Boltzmann function can be fitted to the pressure- and temperature-dependent sigmoidal curve progression of the secondary structural elements.
The pressure- and temperature-induced intensity changes are given by

$$
\begin{gathered}
I=\frac{I_{\mathrm{f}}-I_{\mathrm{u}}}{1+\mathrm{e}^{-\left(\frac{1}{T_{\mathrm{u}}}-\frac{1}{T}\right) \cdot \frac{\Delta H_{\mathrm{vH}, \mathrm{u}}}{R}}}+I_{\mathrm{u}} \\
I=\frac{I_{\mathrm{f}}-I_{\mathrm{u}}}{1+\mathrm{e}^{-\left(p-p_{\mathrm{u}}\right) \cdot \frac{\Delta V_{\mathrm{u}}}{R T}}}+I_{\mathrm{u}}
\end{gathered}
$$

where $I_{\mathrm{f}}$ and $I_{\mathrm{u}}$ are the plateau values of the IR band intensities of the folded and unfolded/dissociated protein, respectively. $T_{\mathrm{u}}$ and $p_{\mathrm{u}}$ (unfolding temperature and pressure) were obtained from the inflection point of the sigmoidal curves. Furthermore, the thermodynamic parameters $\Delta H_{\mathrm{vH}, \mathrm{u}}$ and $\Delta V_{\mathrm{u}}$ (van't Hoff enthalpy and volume changes) can be directly determined from the fits of the temperature- and pressure-dependent experimental data, respectively.

\subsection{Small-angle X-ray scattering (SAXS)}

SAXS measurements at ambient pressure at $25{ }^{\circ} \mathrm{C}$ were carried out on an Anton Paar SAXSess $\mathrm{mc}^{2}$ (Graz, Austria) using a monochromatic X-ray beam $(\lambda=1.54 \AA)$ and an image plate detector. A protein concentration of $15 \mathrm{mg} \mathrm{mL}^{-1}$ with a volume of $20 \mu \mathrm{L}$ was loaded into the quartz capillary ( $\mu$ Cell of Anton Paar). Scattering patterns were collected over $30 \mathrm{~min}$ per image and the data were treated and analysed with 2D-SAXSquant and SAXS Quant. Further, the background of the capillary and buffer was subtracted from the data and the scattering curves were desmeared with the scattering length profile. Pressuredependent (SAXS) measurements were performed at beamline BL9 of the synchrotron radiation facility DELTA (Dortmund, Germany) using a home-built high-pressure sample cell with flat diamond windows. ${ }^{27-29}$ The energy of the X-ray was $10 \mathrm{keV}$, corresponding to a wavelength of $\lambda=1.2 \AA$. An image plate detector MAR345 $(d=345 \mathrm{~mm}, 100 \mu \mathrm{m}$ pixel size) was exposed 15 min per measurement in the pressure range from 1 to $3500 \mathrm{bar}$ at $50{ }^{\circ} \mathrm{C}$. Silver behenate was used to calibrate the momentum transfer $Q$ of the X-rays, which is given by $Q=(4 \pi / \lambda) \sin \theta$, where $\lambda$ is the wavelength of the X-rays and $2 \theta$ is the scattering angle. The GroEL-GroES complex (molar ratio of 1:2) was prepared freshly in $50 \mathrm{mM}$ Tris buffer containing $10 \mathrm{mM} \mathrm{MgCl}_{2}, 100 \mathrm{mM}$ $\mathrm{KCl}$ and $3 \mathrm{mM}$ ATP. Here, the data were processed with fit2D, matlab and SAXSutilities. ${ }^{30}$ The pair distance distribution function, $p(r)$, which depends on the molecular particle shape and on the intra-particle scattering distribution, is given by the indirect Fourier transform of the measured scattered intensity, $I(Q)$. For a particle of uniform electron density, it is given by

$$
p(r)=\frac{1}{2 \pi^{2}} \int_{0}^{\infty} I(Q) Q r \sin (Q r) \mathrm{d} Q
$$

The function $p(r)$ represents the frequency of vector length $r$ connecting small volume elements within the volume of the scattering particle, that is, the protein molecule, with maximum dimension $D_{\max }$, and allows to characterize the size and shape of the scattering particle. The $p(r)$ function 
was calculated by the method of Svergun using the ATSAS software package. ${ }^{31}$

\section{Results and discussion}

\subsection{Small-angle X-ray scattering (SAXS) measurements}

SAXS measurements were employed to verify the correct structure of GroEL, GroES and the whole chaperonin system, GroEL + GroES (at molar ratio $1: 2$, the ratio within E. coli cells) (Fig. 2a and b). Fig. 2a shows the SAXS intensity profiles, $I(Q)$, and (c) the corresponding pair-distance distribution functions, $p(r)$, of GroEL, GroES and the GroEL-GroES complex in the presence of $3 \mathrm{mM}$ ATP in aqueous buffer solution at ambient temperature and pressure. The scattering patterns measured agree very well with literature data, ${ }^{32}$ confirming the correct structures of the proteins and the monodispersity of the samples. The maximal dimension, $D_{\max }$, of GroES is about $10 \mathrm{~nm}$, and that of the GroEL-GroES complex about 17.5 to $20 \mathrm{~nm}$ (Fig. 2c and d), in good agreement with literature data for the asymmetric chaperonin complex, which is known to be prevalent under these and also under physiological conditions. ${ }^{32}$

Fig. $2 \mathrm{~b}$ and $\mathrm{d}$ show the SAXS profiles of the GroEL-GroES complex in the presence of $3 \mathrm{mM}$ ATP as a function of hydrostatic pressure and the corresponding pair distance distribution functions, $p(r)$. As can be clearly seen, the minima of the $I(Q)$ curve, which are typical for the shape of the chaperonin complex, become less pronounced upon compression beyond $2 \mathrm{kbar}$, and have finally disappeared at $3.5 \mathrm{kbar}$. The concomitant decrease of the scattering intensity with increasing pressure is due to a pressure-induced decrease of the electron density contrast between the sample and the solvent, which is due to a drastic increase of the electron density of the water at high pressures. In agreement with the marked changes observed in the $p(r)$ function, these results indicate dissociation of the GroEL-GroES complex at pressures beyond about 2 kbar, which would be consistent with conclusions based on indirect measurements using light scattering methodology. ${ }^{21}$ Even above $3.5 \mathrm{kbar}$, no complete dissociation into monomers is observed, as can be deduced from the slope of $I(Q)$ and shape of the $p(r)$ function, which can be attributed to expanded 2D arrays of protein assemblies.

\subsection{Temperature- and pressure-dependent FTIR spectroscopic measurements}

To reveal also the secondary structural changes of the chaperonin system over a wide range of temperatures and pressures, FTIR spectroscopy was employed. Fig. 3 shows the effect of temperature on the amide I' band of GroEL and GroES in neat buffer and their corresponding changes in secondary structure elements. Details of the assignment and band fitting procedure to determine the various secondary structure elements are illustrated in Fig. S1, ESI. $\dagger$ The normalized amide $\mathrm{I}^{\prime}$ band of GroEL as a function of temperature (Fig. 3a) shows a broad band at $\sim 1650 \mathrm{~cm}^{-1}$, which decreases with increasing temperature, while two peaks appear at $\sim 1618 \mathrm{~cm}^{-1}$ and $\sim 1683 \mathrm{~cm}^{-1}$, respectively.
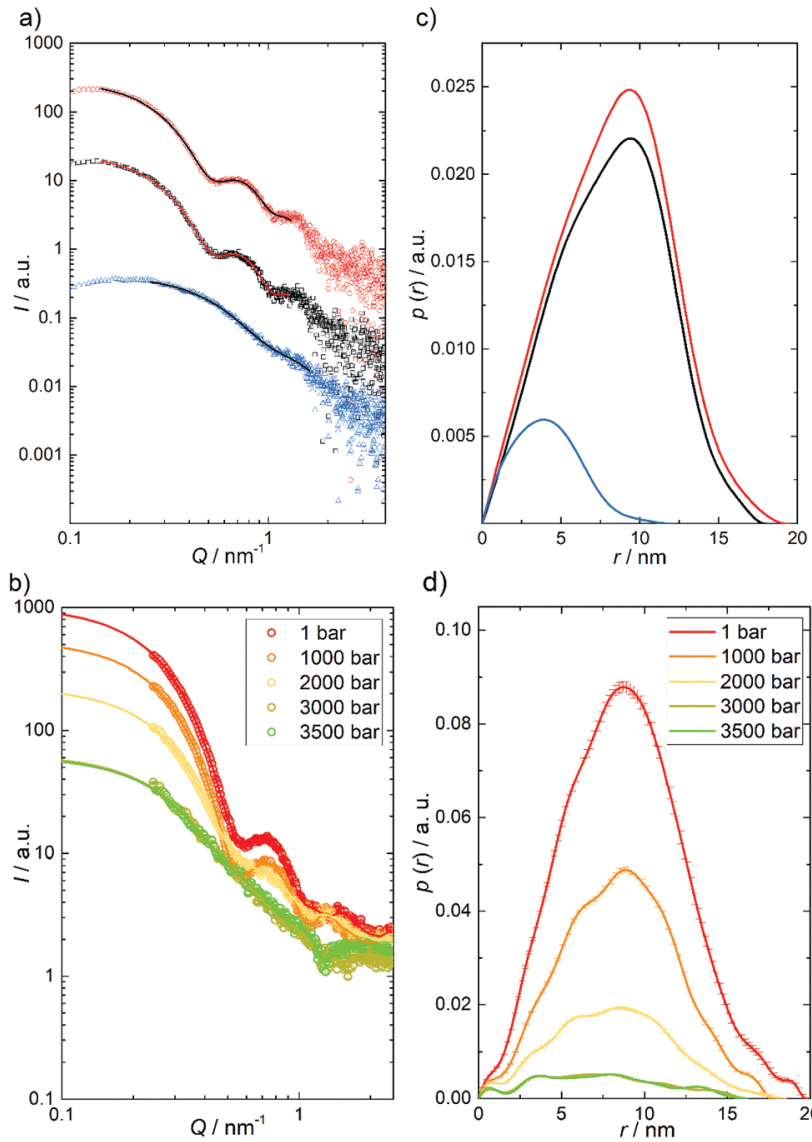

d)

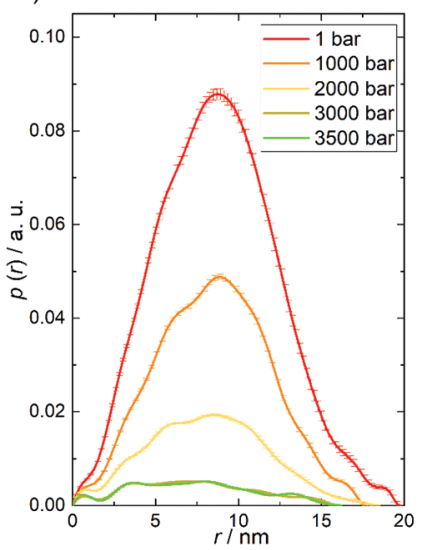

Fig. 2 (a) SAXS intensity curves, $I(Q)$, and (c) the pair-distance distribution functions, $p(r)$, of GroEL (15 mg mL $\mathrm{mb}^{-1}$, black), GroES (15 mg mL $\mathrm{mL}^{-1}$, blue) and the GroEL-GroES complex (molar ratio of $1: 2$ ) in the presence of $3 \mathrm{mM}$ ATP (red) in aqueous solution $(50 \mathrm{mM}$ Tris buffer, $100 \mathrm{mM} \mathrm{KCl}, 10 \mathrm{mM}$ $\left.\mathrm{MgCl}_{2}, \mathrm{pH} 7.5\right)$ at room temperature $\left(T \approx 25^{\circ} \mathrm{C}\right)$. (b) Pressure-dependent SAXS curves and (d) the pair-distance distribution functions, $p(r)$, of the GroEL-GroES complex (25 mg mL $\mathrm{mg}^{-1}$ GroEL, $4.5 \mathrm{mg} \mathrm{mL}^{-1}$ GroES) in aqueous solution $(50 \mathrm{mM}$ Tris buffer, $100 \mathrm{mM} \mathrm{KCl}, 10 \mathrm{mM} \mathrm{MgCl}$ and $3 \mathrm{mM}$ ATP, $\mathrm{pH}$ 7.5). Symbols show experimental data and solid lines in (a) are fits representing the Fourier-transformations of $p(r)$. Owing to strong background scattering by the high-pressure cell and the smaller $Q$-range covered, the $p(r)$ data in Fig. $2 \mathrm{~d}$ appear to be a bit noisier compared to those measured in quartz capillaries at ambient conditions (Fig. 2b).

These two bands are characteristic for aggregation upon unfolding of proteins at high temperature and can be assigned to intermolecular $\beta$-sheets. Based on the fitting procedure, the main secondary structural components at $25{ }^{\circ} \mathrm{C}$ and ambient pressure are $\alpha$-helices $(\sim 39 \%)$, in agreement with literature values, ${ }^{33}$ and intramolecular $\beta$-sheets $(\sim 23 \%)$. These results are in rather good agreement with crystallographic data obtained by Braig et al., ${ }^{34}$ who found $\sim 45 \% \alpha$-helices and $\sim 17 \%$ intramolecular $\beta$-sheets. The small differences observed might be due to different absorption coefficients of the secondary structure elements. Here, we consider and discuss essentially relative changes, only. The amide $\mathrm{I}^{\prime}$ band shows no significant changes between 25 and $65{ }^{\circ} \mathrm{C}$, where thermal unfolding sets in, which is accompanied by decreasing amounts of $\alpha$-helices $(\sim-10 \%)$ and random coils $(\sim-7 \%)$ 
a)

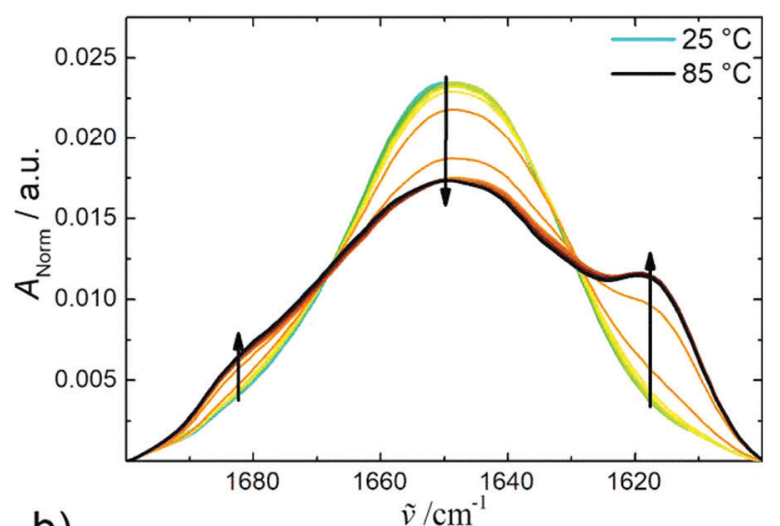

b)

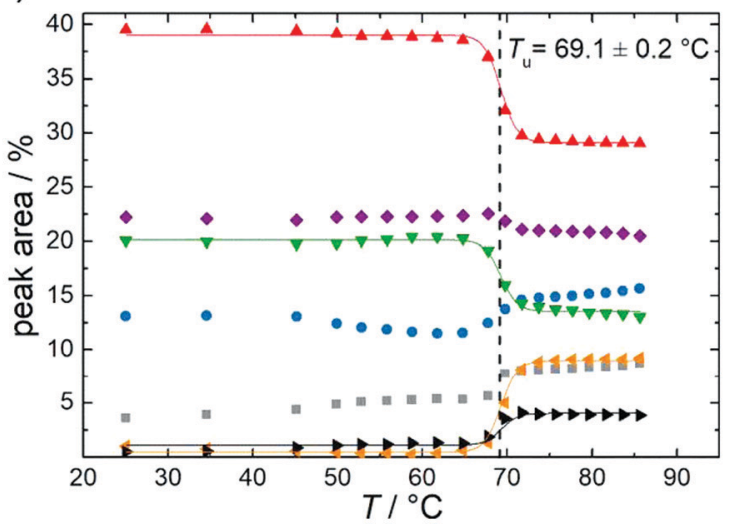

c)

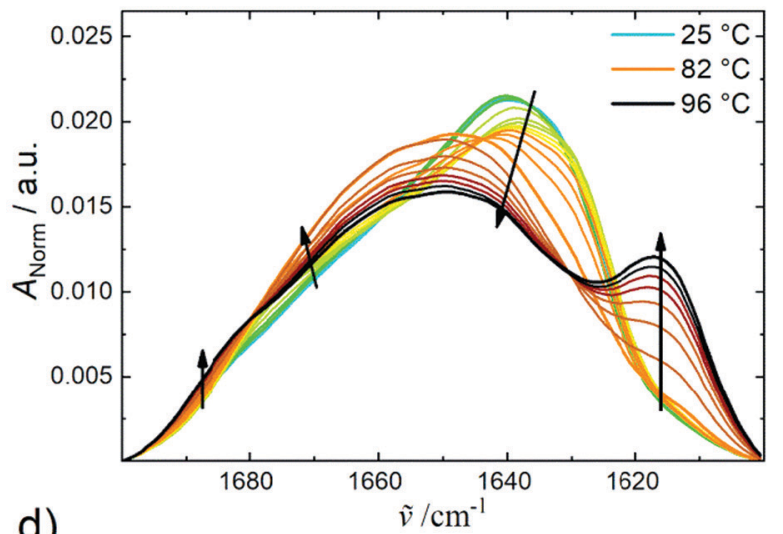

d)

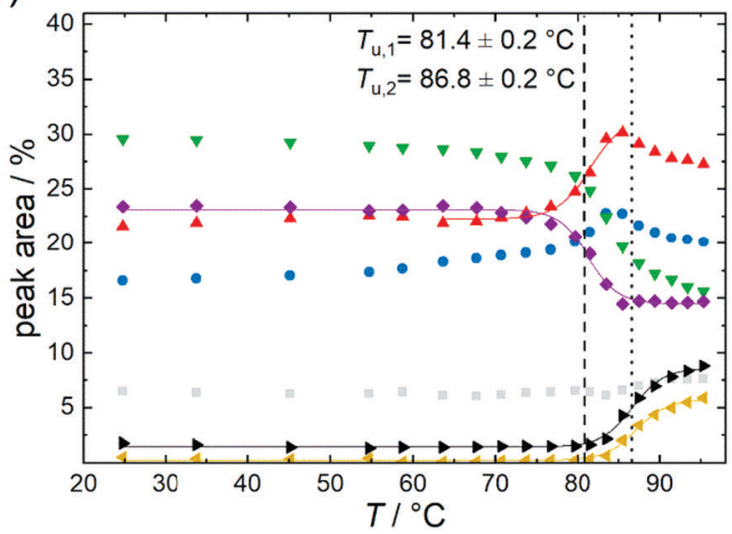

Fig. 3 Temperature-dependent FTIR absorption data of GroEL and GroES in neat buffer at ambient pressure. (a) Temperature-dependent changes of the normalized amide I' band region of GroEL and (b) corresponding changes in secondary structure elements by temperature: intermolecular $\beta$-sheets $\left(1683 \mathrm{~cm}^{-1}\right.$, grey); turns and loops $\left(1672 \mathrm{~cm}^{-1}\right.$, blue); $\alpha$-helices $\left(1654 \mathrm{~cm}^{-1}\right.$, red); random coils $\left(1642 \mathrm{~cm}^{-1}\right.$, green); intramolecular $\beta$-sheets $\left(1636 \mathrm{~cm}{ }^{-1}\right.$, purple); intermolecular $\beta$-sheets $\left(1618 \mathrm{~cm}^{-1}\right.$, orange) and side chains $\left(1612 \mathrm{~cm}^{-1}\right.$, black). (c) Temperature-dependent changes of the normalized amide $\mathrm{I}^{\prime}$ band region of GroES and (d) corresponding changes in secondary structure elements by temperature: intermolecular $\beta$-sheets $\left(1685 \mathrm{~cm}^{-1}\right.$, grey); turns and loops $\left(1672 \mathrm{~cm}^{-1}\right.$, blue); $\alpha$-helices $\left(1654 \mathrm{~cm}^{-1}\right.$, red); random coils $\left(1641 \mathrm{~cm}^{-1}\right.$, green); intramolecular $\beta$-sheets $\left(1630 \mathrm{~cm}{ }^{-1}\right.$, purple); intermolecular $\beta$-sheets $\left(1618 \mathrm{~cm}^{-1}\right.$, orange) and side chains $\left(1613 \mathrm{~cm}^{-1}\right.$, black). Lines display the Boltzmann fits to the experimental data using eqn (1).

and, concomitantly, an increasing amount of intermolecular $\beta$-sheets $(\sim+13 \%)$. Boltzmann fits to the experimental data (eqn (1)) allow to determine the unfolding temperature, $T_{\mathrm{u}}$, and the van't Hoff enthalpy change, $\Delta H_{\mathrm{vH}, \mathrm{u}}$, upon (partial) unfolding of the protein: $T_{\mathrm{u}}=69.1 \pm 0.2{ }^{\circ} \mathrm{C}$ and $\Delta H_{\mathrm{vH}, \mathrm{u}}=1000 \pm 60 \mathrm{~kJ} \mathrm{~mol}^{-1}$ (Table SI 1). The unfolding temperature is in accord with the literature, which reports denaturation of GroEL to occur between

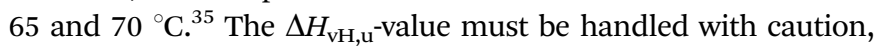
however, due to the irreversibility of the heat-induced protein unfolding and subsequent aggregation process, i.e., it reflects no true thermodynamic data and serves only for the comparison with $\Delta H_{\mathrm{vH}, \mathrm{u}}$ values determined in the presence of different salts, nucleotides and cosolvents (see below).

In contrast to GroEL, the normalized amide I' band of GroES shows a broad band at $\sim 1640 \mathrm{~cm}^{-1}$ (Fig. 3c), which indicates a different distribution of secondary structure elements. Upon increasing temperature, we observe, on the one hand, a decrease of the main peak and on the other hand, a distinct structural transition (turquoise to orange line) before aggregation commences, which is indicated by the intermolecular $\beta$-sheet peaks appearing at $\sim 1617 \mathrm{~cm}^{-1}$ and $\sim 1684 \mathrm{~cm}^{-1}$. GroES shows high contents of random coils $(\sim 29 \%)$ and intramolecular $\beta$-sheets $(\sim 23 \%)$ at $25{ }^{\circ} \mathrm{C}$ and ambient pressure, and the amount of $\alpha$-helices is much lower than in GroEL. Valencia et al. reported similar values for the secondary structure elements. ${ }^{36}$ The secondary structure of GroES does not exhibit significant changes between 25 and $70{ }^{\circ} \mathrm{C}$. Beyond $70{ }^{\circ} \mathrm{C}$, a structural transition involving intramolecular $\beta$-sheets transforming to more $\alpha$-helices takes place. The aggregation of the protein starts at $\sim 82{ }^{\circ} \mathrm{C}$, where the percentage of intermolecular $\beta$-sheets increases to $7 \%$, while the percentage of $\alpha$-helices decreases slightly ( $\sim-4 \%)$. During the overall phase transition, a continuous decrease of the percentage of random coils $(\sim-12 \%)$ is observed. The temperatures of the two structural transitions are located at $T_{\mathrm{u}, 1}=81.4 \pm 0.2{ }^{\circ} \mathrm{C}$ and $T_{\mathrm{u}, 2}=86.8 \pm 0.2{ }^{\circ} \mathrm{C}$, respectively. Hence, GroES displays an about $12{ }^{\circ} \mathrm{C}$ higher temperature stability than GroEL. As shown by Boudker et al., the stability of GroES seems to be concentration-dependent, because the $T_{\mathrm{u}}$-value was found to increase with increasing protein concentration. ${ }^{37}$ DSCand CD-experiments revealed a $T_{\mathrm{u}}$-value of about $76.4{ }^{\circ} \mathrm{C}$ for a 
a)

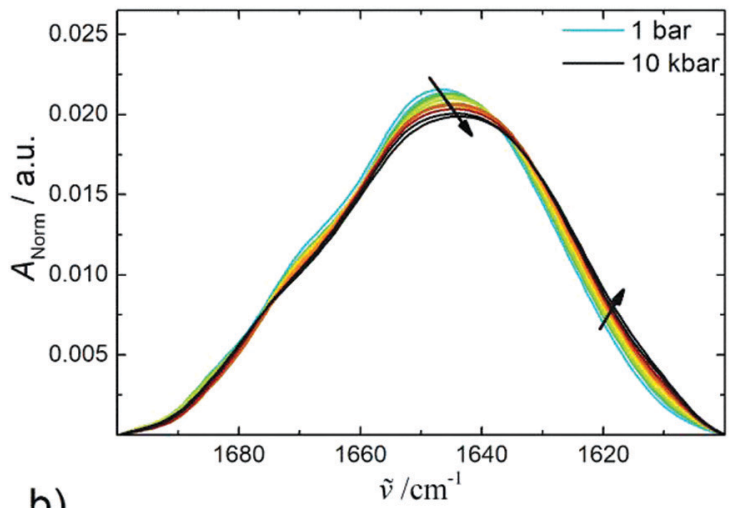

b)

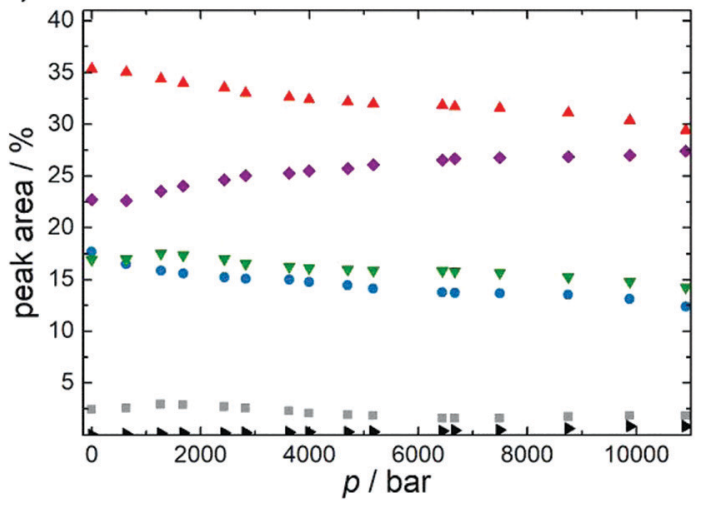

c)
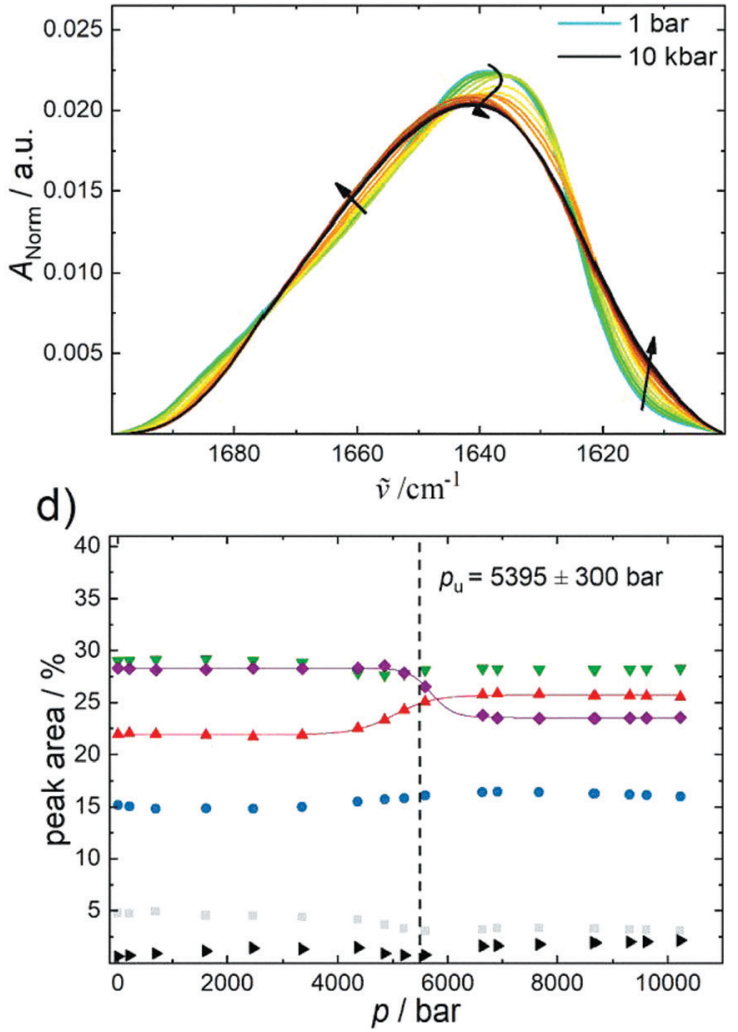

Fig. 4 Pressure-dependent FTIR absorption data of GroEL and GroES in neat buffer at $25^{\circ} \mathrm{C}$. (a) Pressure-dependent changes of the normalized amide $\mathrm{I}^{\prime}$ band region of GroEL and (b) corresponding changes in secondary structure elements by pressure: intermolecular $\beta$-sheets (1683 $\mathrm{cm}^{-1}$, grey); turns and loops $\left(1672 \mathrm{~cm}^{-1}\right.$, blue); $\alpha$-helices $\left(1654 \mathrm{~cm}^{-1}\right.$, red); random coils $\left(1641 \mathrm{~cm}^{-1}\right.$, green); intramolecular $\beta$-sheets $\left(1635 \mathrm{~cm}^{-1}\right.$, purple) and side chains (1611 $\mathrm{cm}^{-1}$, black). (c) Pressure-dependent changes of the normalized amide I' band region of GroES and (d) corresponding changes in secondary structure elements by pressure: intermolecular $\beta$-sheets $\left(1683 \mathrm{~cm}^{-1}\right.$, grey); turns and loops $\left(1671 \mathrm{~cm}^{-1}\right.$, blue); $\alpha$-helices $\left(1654 \mathrm{~cm}{ }^{-1}\right.$, red); random coils $\left(1641 \mathrm{~cm}^{-1}\right.$, green); intramolecular $\beta$-sheets $\left(1630 \mathrm{~cm}^{-1}\right.$, purple) and side chains $\left(1612 \mathrm{~cm}^{-1}\right.$, black). Lines display Boltzmann fits to the experimental data using eqn (2).

GroES concentration of $175 \mu \mathrm{M}$. Here, we used a much higher GroES concentration, $\sim 1.44 \mathrm{mM}$.

Fig. 4 highlights the effect of pressure on the amide $\mathrm{I}^{\prime}$ band and the fraction of secondary structures of GroEL and GroES derived from the analysis of the underlying subbands (Fig. SI2, ESI $\dagger$ ). For both proteins, a pressure range between 1 bar and 10000 bar $(1 \mathrm{GPa})$ has been covered. The amide $\mathrm{I}^{\prime}$ band of GroEL is shifting towards lower wavenumbers upon application of pressure (Fig. 4a). This linear pressure-induced elastic shift can be explained by a pressureinduced compression of the chemical bonds, equivalent to changes of the force constant of the $\mathrm{C}=\mathrm{O}$ stretching vibration.

Regarding the percentage of secondary structure elements, a slightly reduction of the $\alpha$-helix content $(\sim-5 \%)$ is observed with increasing pressure, whereas the content of intramolecular $\beta$-sheets increases concomitantly $(\sim+5 \%)$ beyond about 1 kbar. Up to $10 \mathrm{kbar}$, only this small conformational change takes place, which is accompanied by a volume change as small as $\Delta V_{\mathrm{u}}=-8.8 \pm 3 \mathrm{~mL} \mathrm{~mol}^{-1}$ as determined using eqn (2). The pressure dependence of the amide $I^{\prime}$ band and relative changes of secondary structure elements of the GroEL-GroES complex are similar to those of GroEL (Fig. SI 3, ESI $\dagger$ ).
The pressure dependence of the secondary structure of GroES differ significantly from that of GroEL. GroES undergoes partial unfolding at $p_{\mathrm{u}}$ of $5.4 \pm 0.3 \mathrm{kbar}$ (Fig. $4 \mathrm{c}$ and d), which is accompanied by an increase of $\alpha$-helices $(\sim+4 \%)$, whereas the content of intramolecular $\beta$-sheets decreases concomitantly ( $\sim-5 \%$ ) upon compression. Using eqn (2), the volume change of partial unfolding of GroES is determined to be $\Delta V_{\mathrm{u}}=$ $-53 \pm 12 \mathrm{~mL} \mathrm{~mol}^{-1}$. This value is typical for $\Delta V_{\mathrm{u}}$-values found for unfolding of monomeric proteins..$^{38-41}$

By favoring states with a smaller partial molar volume, pressure generally shifts an equilibrium toward a state with smaller overall volume, in accord with Le Châtelier's principle. ${ }^{16,41}$ Hence, owing to the fact that the pressure stability of proteins in general, and of oligomeric proteins in particular, is largely due to their internal void volume and packing defects, which get filled with water upon unfolding, ${ }^{38}$ we can conclude that the packing of the two proteins at ambient temperature is different. But the difference is very small, comparable to the volume of just 2-3 water molecules, only. GroEL exhibits a small $\Delta V_{\mathrm{u}}$-value of about $-8.8 \mathrm{~mL} \mathrm{~mol}^{-1}$ and experiences small conformational changes above 1 kbar only, where the amount of intramolecular $\beta$-sheets increases slightly at the expense 
of $\alpha$-helices, whereas GroES tolerates pressures up to about $5.4 \mathrm{kbar}$, where partial unfolding of the protein commences, which is accompanied by a volume change of about $-53 \mathrm{~mL} \mathrm{~mol}^{-1}$.

\subsection{Effects of cosolvents, salts and nucleotides on the folding stability of the chaperonin system}

Cations, nucleotides and osmolytes have been shown to be important for the maintenance of the reaction cycle of the chaperonin system and for osmotic pressure regulation. ${ }^{42,43}$ Hence, studies of the effects of salts, ATP, ADP, and of TMAO on the thermo- and piezostability of GroEL, GroES and the GroEL-GroES complex have been included in our studies using FTIR spectroscopy. All results obtained are depicted in Fig. 5a and are listed in Table SI 1 (ESI $\dagger$ ). In the presence of $100 \mathrm{mM} \mathrm{K}^{+}$ (buffer B) and additionally $1 \mathrm{M}$ TMAO, the unfolding temperature of GroEL is significantly shifting towards higher temperatures $\left(\Delta T_{\mathrm{u}} \approx 5^{\circ} \mathrm{C}\right)$, i.e. these cosolutes increase the stability of the proteins. Addition of $3 \mathrm{mM}$ of the nucleotide ADP and addition of $1 \mathrm{M}$ urea show the opposite effect. Differently, GroES experiences no significant changes of the $T_{\mathrm{u}}$-value in the presence of salts and nucleotides. However, the unfolding temperature of GroES is found to be highly dependent on the cosolvents TMAO and urea, which also have a positive (1 M TMAO) or negative ( $1 \mathrm{M}$ urea) effect on the temperature stability of the protein. Interestingly, the stability of GroEL is also enhanced by addition of GroES (molar ratio of 1:2), in good agreement with the literature, ${ }^{11}$ both in the absence and presence of cosolvents (Fig. 5a). The spectroscopically determined van't Hoff enthalpy changes (Table SI 1, ESI $\dagger$ ) are not significantly affected by the addition of salts, nucleotides and TMAO. The effect of salts and nucleotides on the thermostability of neat GroEL are in good agreement with data derived from differential scanning calorimetry by Surin et al. ${ }^{11}$ The destabilizing effect of the nucleotide can be explained by conformational changes in the open state of the GroEL subunit structure, which occur probably near the nucleotide binding site. ${ }^{44,45}$ Also urea exhibits a negative influence on the stability, which results most likely from direct interactions of the urea molecules with the protein backbone and its side chains. Hence, the unfolded state is energetically favored and stabilized relative to the native conformation. ${ }^{46,47}$ In contrast, TMAO is able to enhance the protein stability without direct interaction with the protein. It prevents unfolding by favorable interaction with water, leading to an increase in hydrogen bonding and structuring of the solvent. ${ }^{40,48}$ Such preferential exclusion effect of TMAO from the protein interface leads to an increase of the stability of folded and compact structures. ${ }^{48,49}$ The presence of $\mathrm{K}^{+}$ions as well as GroES also affect the temperature stability in a positive way. Both factors regulate and stabilize the quaternary structure of GroEL. GroES interacts with the apical domain of $\mathrm{GroEL}_{197-376}$ and triggers a conformational change. ${ }^{24,50,51} \mathrm{~K}^{+}$and $\mathrm{Mg}^{2+}$ ions play also an important role for ATP binding and hydrolysis and have an impact on the stability of GroEL. ${ }^{52-54}$

Temperature-induced unfolding of proteins is mainly driven by the conformational entropy gain of the peptide chain, whereas the overall volume change plays the decisive role in the a)
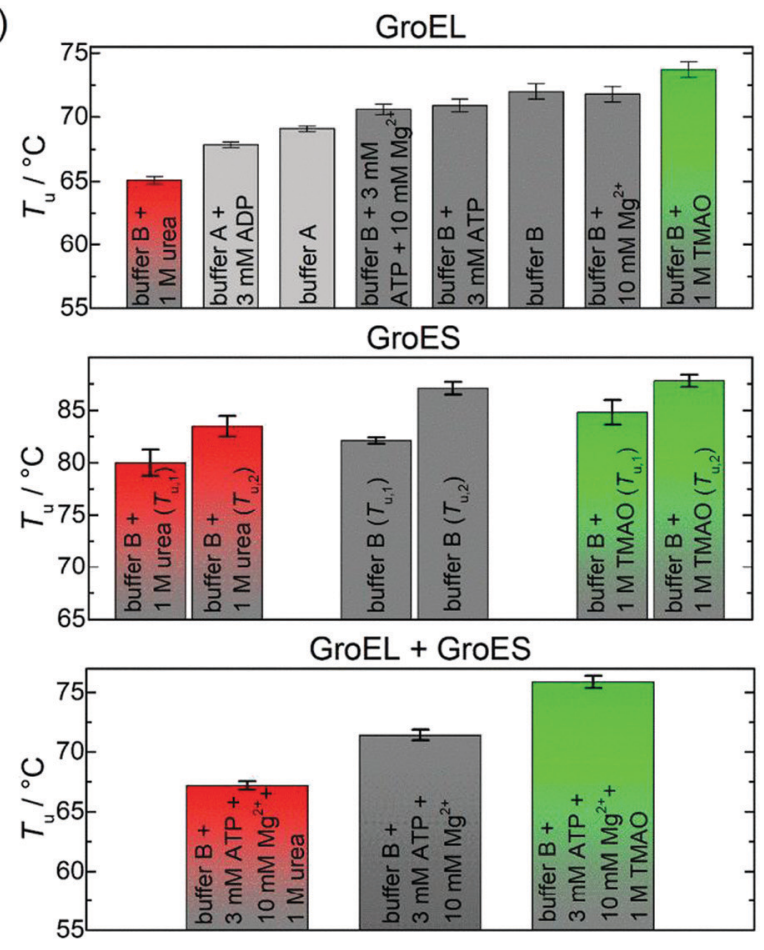

b)
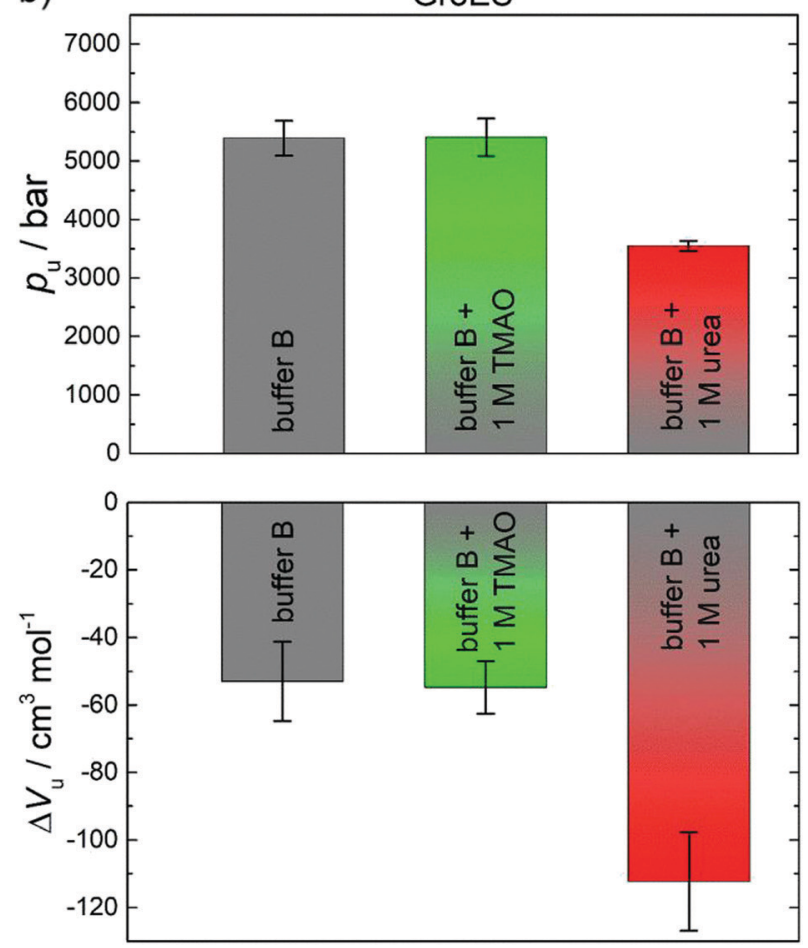

Fig. 5 (a) Spectroscopically determined unfolding temperatures, $T_{u}$, of GroEL, GroES and the whole complex at ambient pressure in the absence and presence of different salt ions, nucleotides and/or cosolvents. The dashed lines show the $T_{u}$-value without GroES for comparison. (b) Spectroscopically determined unfolding pressures, $p_{u}$, and volume changes, $\Delta V_{u}$, of GroES at $25^{\circ} \mathrm{C}$ in the absence and presence of different cosolvents. Here, buffer $A$ is the neat buffer system, while buffer $B$ contains additional $100 \mathrm{mM} \mathrm{KCl}$. 
pressure-induced unfolding scenario. ${ }^{16,41}$ The system tends to occupy a smaller volume state at high pressure, which includes the filling and elimination of internal void volume and cavities as possible mechanism for pressure-induced unfolding. ${ }^{38,41,55}$ The thermodynamic data for GroES in the presence of the two cosolvents studied compared to the neat buffer data are displayed in Fig. $5 \mathrm{~b}$ and Table SI 2 (ESI $\dagger$ ). Within the accuracy of the experiment, $1 \mathrm{M}$ TMAO has no significant effect on $p_{\mathrm{u}}$ and $\Delta V_{\mathrm{u}}$. A similar behavior has been observed for GroEL (data not shown).

Differently, marked stabilizing effects of some monomeric proteins (e.g., SNase, RNase A) by TMAO against pressureinduced unfolding and favorable effects of TMAO on enzyme function, protein polymerization and channel activity under high-pressure stress have been reported. ${ }^{41,56-63}$ The minor (possibly stabilizing) effect observed here is probably due to a smaller degree of unfolding and exposure of solvent accessible surface area (SASA), rendering the excluded volume effect imposed by TMAO less effective.

The marine-osmolyte TMAO has been discussed as a potential piezolyte, i.e. an osmolyte-type solute that is able to counteract deteriorating pressure effects, in many deep sea vertebrate animals (e.g., chondrichthyans (such as sharks and rays), teleost fishes, crustaceans), which in addition has the ability to counteract destabilizing effects of urea. ${ }^{17,60}$ In deep sea invertebrates, cells contain high levels of neutral free amino acids and some other methylamines, ${ }^{64}$ which show different cytoprotective properties against environmental stressors. For example, hypotaurine seems to protect cells as an antioxidant from sulfide toxicity. ${ }^{18}$ Glycine, in comparison to TMAO, appears to stabilize proteins essentially against temperature stress. ${ }^{60}$

Conversely, $p_{\mathrm{u}}$ markedly decreases by $\sim 1800$ bar in the presence of $1 \mathrm{M}$ urea and, consequently, $\Delta V_{\mathrm{u}}$ is much more negative compared to the cosolvent-free buffer (buffer B). The pressureinduced destabilization of urea is expected to be due to favorable enthalpic interactions of urea with the protein, leading to marked conformational changes. They may lead to an increase of void volume and/or an increase of electrostrictive hydration of a larger exposed SASA upon (partial) unfolding, and hence to the more negative $\Delta V_{\mathrm{u}}$-value observed. ${ }^{41,59}$

\section{Conclusions}

The chaperonin system GroEL-GroES is present in all kingdoms of life and rescues proteins from improper folding and aggregation. As a usual answer to a stress condition, including high temperatures and pressures, production of heat shock proteins such as the GroEL-GroES system, is boosted. ${ }^{13,65,66}$ Here, we explored the thermo- and piezostability of GroEL, GroES and the GroEL-GroES complex itself, covering a large region of the temperature-pressure plane, and included the effects of particular cosolvents, nucleotides and salts, employing quantitative FTIR spectroscopy, complemented by SAXS data. Owing to its high biological relevance and lack of data, our focus was especially on the effect of pressure on the chaperonin system. The experimental results reveal that the GroEL-GroES complex is remarkably temperature stable with an unfolding temperature beyond $\sim 70{ }^{\circ} \mathrm{C}$, which can still be slightly increased by compatible cosolutes like TMAO, facilitated by the preferential exclusion of TMAO from the protein surface. Conversely, the pressure stability of GroEL and hence the GroEL-GroES complex is rather limited and much less than that of monomeric proteins, which typically unfold beyond pressures of 4-8 kbar, only. ${ }^{13,17}$ Whereas GroES is stable up to about 5.5 kbar before it partially unfolds, GroEL and hence the GroEL-GroES complex undergoes minor conformational changes already beyond $1 \mathrm{kbar}$, which can be attributed to a dissociation-induced conformational drift, leading to a slight decrease of the $\alpha$-helix content and a concomitant increase of intramolecular $\beta$-sheets. The FTIR data are corroborated by the high-pressure SAXS data, which reveal dissociation of the complex at about 2 kbar. Such conclusions would be in line with early light scattering experiments by Horowitz et al., suggesting dissociation of the system at pressures around $2 \mathrm{kbar}$, and the reassociation of the complex being very slow with half-times of several days, which might be due to the conformational drift of the structure upon dissociation of the complex. ${ }^{67}$ Further, the FTIR data show, quite unexpectedly, that no significant unfolding of GroEL is observed even up to $10 \mathrm{kbar}$, i.e., the subunits are very pressure stable themselves. The SAXS data measured up to $3.5 \mathrm{kbar}$ also indicate that no complete dissociation into the small monomeric subunits and unfolding of the monomers takes place. Accumulation of particular cosolutes, such as methylamines, polyols and amino acids, are often found in cells to equilibrate cellular osmotic pressure and to maintain stability and functionality of proteins and their assemblies. ${ }^{17-20,40}$ Therefore, such small organic molecules are termed osmolytes or chemical chaperones. A prominent and effective osmolyte, trimethylamine- $N$-oxide (TMAO), is even found in tissue of various deep sea animals and its concentration correlates with the ocean depth, i.e. hydrostatic pressure, where those animals were encountered. ${ }^{17,18,60}$ Here we show that $1 \mathrm{M}$ TMAO is able to increase the temperature stability of the proteins slightly, the pressure stability is hardly affected by the cosolute, only, which might be due to the absence of significant unfolding of the subunits.

Hence, overall, the GroEL-GroES complex is rather temperature stable and stable against high hydrostatic pressure in the range of 1 to about 1000 bar, where dissociation of the GroELGroES complex sets in. Thus, the chaperonin system is just stable in the pressure range encountered to life on Earth, including the deepest trenches of the ocean. It remains to be learned, how cells of bacteria and archaea are able to withstand more elevated temperature and pressure conditions as encountered in $\mathrm{km}$ depths of the sub-seafloor crust. ${ }^{15}$

\section{Conflicts of interest}

The authors declare no conflicts of interest.

\section{Acknowledgements}

Diese Arbeit wurde durch RESOLV unterstützt durch die Deutsche Forschungsgemeinschaft (DFG) im Rahmen der Exzellenzstrategie 
des Bundes und der Länder - EXC2033 - Projektnummer 390677874. Financial support from the DFG Research Unit FOR 1979 is also gratefully acknowledged. We thank Dr Manajit Hayer-Hartl and her group (MPI Biochemistry, Germany) for providing GroEL and GroES plasmids. The authors thank DELTA for experimental time at beamline BL9. Special thanks go to Dr M. Paulus and Dr C. Sternemann for their support at the beamline and G. Surmeier with the high-pressure setup. We are grateful for experimental help by M. Manisegaran, J.-M. Knop and Dr I. Kiesel.

\section{References}

1 D. Balchin, M. Hayer-Hartl and F. U. Hartl, Science, 2016, 353, aac4354.

2 F. Hartl and M. Hayer-Hartl, Sci. Compass, 2002, 295, 1852-1858.

3 A. Cario, M. Jebbar, A. Thiel, N. Kervarec and P. M. Oger, Sci. Rep., 2016, 6, 1-8.

4 F. U. Hartl, A. Bracher and M. Hayer-Hartl, Nature, 2011, 475, 324-332.

5 A. J. Gupta, S. Haldar, G. Miličić, F. U. Hartl and M. HayerHartl, J. Mol. Biol., 2014, 426, 2739-2754.

6 S. Sharma, K. Chakraborty, B. K. Müller, N. Astola, Y. C. Tang, D. C. Lamb, M. Hayer-Hartl and F. U. Hartl, Cell, 2008, 133, 142-153.

7 H. Taguchi, J. Biochem., 2005, 137, 543-549.

8 W. A. Fenton and A. L. Horwich, Q. Rev. Biophys., 2003, 36, 229-256.

9 D. Thirumalai and G. H. Lorimer, Annu. Rev. Biophys. Biomol. Struct., 2001, 30, 245-269.

10 A. Marx and E. Mandelkow, Eur. Biophys. J., 1994, 22, 405-421.

11 A. K. Surin, N. V. Kotova, I. A. Kashparov, V. V. Marchenkov, S. Y. Marchenkova and G. V. Semisotnov, FEBS Lett., 1997, 405, 260-262.

12 B. Sot, S. Bañuelos, J. M. Valpuesta and A. Muga, J. Biol. Chem., 2003, 278, 32083-32090.

13 F. Meersman, I. Daniel, D. H. Bartlett, R. Winter, R. Hazael and P. F. McMillan, Rev. Mineral. Geochem., 2013, 75, 607-648.

14 I. Daniel, P. Oger and R. Winter, Chem. Soc. Rev., 2006, 35, 858-875.

15 B. Orcutt and I. Daniel, in DeepCarbon: Past to Present, ed. R. Dasgupta, Cambridge University Press, 2019.

16 K. Akasaka and H. Matsuki, High Pressure Bioscience, Springer, Heidelberg, 2015.

17 R. H. Kelly and P. H. Yancey, Biol. Bull., 1999, 196, 18-25.

18 P. H. Yancey, W. R. Blake and J. Conley, Comp. Biochem. Physiol., Part A: Mol. Integr. Physiol., 2002, 133, 667-676.

19 M. A. Schroer, Y. Zhai, D. C. F. Wieland, C. J. Sahle, J. Nase, M. Paulus, M. Tolan and R. Winter, Angew. Chem., Int. Ed., 2011, 50, 11413-11416.

20 K. Julius, J. Weine, M. Berghaus, N. König, M. Gao, J. Latarius, M. Paulus, M. A. Schroer, M. Tolan and R. Winter, Phys. Rev. Lett., 2018, 121, 038101.

21 M. Panda, J. Ybarra and P. M. Horowitz, J. Biol. Chem., 2001, 276, 6253-6259.
22 M. Panda and P. M. Horowitz, Biochemistry, 2002, 41, 1869-1876. 23 M. Panda, J. Ybarra and P. M. Horowitz, Biochemistry, 2002, 41, 12843-12849.

24 N. A. Ryabova, V. V. Marchenkov, S. Y. Marchenkova, N. V. Kotova and G. V. Semisotnov, Biochemistry, 2013, 78, 1405-1414. 25 P. Wong and D. Moffat, Appl. Spectrosc., 1989, 43, 1279-1281. 26 G. Panick and R. Winter, Biochemistry, 2000, 39, 1862-1869.

27 C. Krywka, M. Paulus, C. Sternemann, M. Volmer, A. Remhof, G. Nowak, A. Nefedov, B. Pöter, M. Spiegel and M. Tolan, J. Synchrotron Radiat., 2006, 13, 8-13.

28 C. Krywka, C. Sternemann, M. Paulus, M. Tolan, C. Royer and R. Winter, ChemPhysChem, 2008, 9, 2809-2815.

29 R. Winter, Biochim. Biophys. Acta, Protein Struct. Mol. Enzymol., 2002, 1595, 160-184.

30 M. Sztucki and T. Narayanan, J. Appl. Crystallogr., 2007, 40, 459-462.

31 D. Franke, M. V. Petoukhov, P. V. Konarev, A. Panjkovich, A. Tuukkanen, H. D. T. Mertens, A. G. Kikhney, N. R. Hajizadeh, J. M. Franklin, C. M. Jeffries and D. I. Svergun, J. Appl. Crystallogr., 2017, 50, 1212-1225.

32 T. Inobe, K. Takahashi, K. Maki, S. Enoki, K. Kamagata, A. Kadooka, M. Arai and K. Kuwajima, Biophys. J., 2008, 94, 1392-1402.

33 J. Chen, H. Yagi, Y. Furutani, T. Nakamura, A. Inaguma, H. Guo, Y. Kong and Y. Goto, Sci. Rep., 2014, 4, 1-7.

34 K. Braig, Z. Otwinowski, R. Hedge, D. C. Boisvert, A. Joachimiak, A. L. Horwich and P. B. Sigler, Nature, 1994, 371, 578-586.

35 J. Martin, A. Horwich and F. Hartl, Science, 1992, 258, 995-998.

36 A. Valencia, T. J. Hubbard, A. Muga, S. Banuelos, O. Llorca, J. L. Carrascosa and J. M. Valpuesta, Proteins: Struct., Funct., Genet., 1995, 22, 199-209.

37 O. Boudker, M. J. Todd and E. Freire, J. Mol. Biol., 1997, 272, 770-779.

38 J. Roche, J. A. Caro, D. N. Norberto, P. B. Barthe, C. R. Roumestand, J. L. Schlessmand, A. E. Garcia, B. GarcíaMoreno and C. A. Royer, Proc. Natl. Acad. Sci. U. S. A., 2012, 109, 6945-6950.

39 J. L. Silva, A. C. Oliveira, T. C. R. G. Vieira, G. A. P. de Oliveira, M. C. Suarez and D. Foguel, Chem. Rev., 2014, 114, 7239-7267. 40 C. A. Royer, Biochim. Biophys. Acta, 2002, 1595, 201-2009.

41 R. Winter, Annu. Rev. Biophys., 2019, 48, 441-463.

42 G. S. Jackson, R. A. Staniforth, D. J. Haisall, T. Atkinson, J. J. Holbrook, A. R. Clarke and S. G. Burston, Biochemistry, 1993, 32, 2554-2563.

43 P. V. Viitanen, T. H. Lubben, J. Reed, P. Goloubinoff, D. P. O'Keefe and G. H. Lorimer, Biochemistry, 1990, 29, 5665-5671.

44 A. Galán, O. Llorca, J. M. Valpuesta, J. Pérez-Pérez, J. L. Carrascosa, M. Menéndez, S. Bañuelos and A. Muga, Eur. J. Biochem., 1999, 259, 347-355.

45 Q. Zhang, J. Chen, K. Kuwajima, H. M. Zhang, F. Xian, N. L. Young and A. G. Marshall, Sci. Rep., 2013, 3, 1247-1253.

46 C. Pace, D. Laurents and J. Thomson, Biochemistry, 1990, 29, 2564-2572.

47 Q. Zou, S. Habermann-Rottinghaus and K. Murphy, Proteins: Struct., Funct., Genet., 1998, 31, 107-115. 
48 D. Canchi and A. García, Annu. Rev. Phys. Chem., 2013, 64, 273-294.

49 D. Canchi, P. Jayasimha, D. Rao, G. Makhatadze and A. Garcia, Biophys. J., 2013, 104, 12095-12104.

50 B. L. De Groot, G. Vriend and H. J. C. Berendsen, J. Mol. Biol., 1999, 286, 1241-1249.

51 A. M. Roseman, S. Chen, H. White, K. Braig and H. R. Saibil, Cell Press, 1996, 87, 241-251.

52 J. P. Grason, J. S. Gresham, L. Widjaja, S. C. Wehri and G. H. Lorimer, Proc. Natl. Acad. Sci. U. S. A., 2008, 105, 17334-17338. 53 J. Wang and D. C. Boisvert, J. Mol. Biol., 2003, 327, 843-855. 54 S. Meena and A. K. Saxena, J. Phys. Chem. Biophys., 2016, 6, 1-6. 55 L. Smeller, Biochim. Biophys. Acta, 2002, 1595, 11-29.

56 L. Arns, V. Schuabb, S. Meichsner, M. Berghaus and R. Winter, Z. Phys. Chem., 2018, 232, 615-634.

57 S. R. Al-Ayoubi, P. H. Schummel, M. Golub, J. Peters and R. Winter, Phys. Chem. Chem. Phys., 2017, 19, 14230-14237. 58 P. H. Schummel, M. W. Jaworek, C. Rosin, J. Högg and R. Winter, Phys. Chem. Chem. Phys., 2018, 20, 28400-28411.
59 T. Q. Luong, S. Kapoor and R. Winter, ChemPhysChem, 2015, 16, 3555-3571.

60 P. H. Yancey and J. F. Siebenaller, J. Exp. Biol., 2015, 218, 1880-1896.

61 A. A. Brindley, R. W. Pickersgill, J. C. Partridge, D. J. Dunstan, D. M. Hunt and M. J. Warren, PLoS One, 2008, 3, e2042.

62 R. R. Petrov, B. Rohde, B. Cornell and B. Martinac, Channels, 2012, 6, 262-271.

63 M. Gao, C. Held, S. Patra, L. Arns, G. Sadowski and R. Winter, ChemPhysChem, 2017, 18, 2951-2972.

64 A. Downing, G. Wallace and P. Yancey, Deep Sea Res., Part I, 2018, 138, 1-10.

65 T. J. Welch, A. Farewell, F. C. Neidhardt and D. H. Bartlett, J. Bacteriol., 1993, 175, 7170-7177.

66 D. H. Bartlett, Biochim. Biophys. Acta, Protein Struct. Mol. Enzymol., 2002, 1595, 367-381.

67 B. Gorovits, C. S. Raman and P. M. Horowitz, J. Biol. Chem., 1995, 270, 2061-2066. 